Guinevere S. Pabayos, MD

Armando M. Chiong Jr., MD

Department of Otolaryngology

Head and Neck Surgery

Ospital ng Maynila Medical Center

\section{Solitary Fibrous Tumor of the Larynx and Anterior Neck}

Whether benign or malignant, laryngeal and neck masses may involve the upper airway and obstruct breathing. While surgically-resectable malignancies are generally extirpated with adequate margins of normal tissue, benign lesions are usually excised conservatively. However, even benign masses may behave malignantly, necessitating more aggressive surgical resection. We present one such case.

\section{CASE REPORT}

A 35-year-old man from Cotabato City consulted due to difficulty of breathing. He had a sixyear history of progressively enlarging anterior neck mass with intermittent dyspnea, foreign body sensation, progressive dysphagia and hoarseness over the last three months. Physical examination revealed a well-defined, $5 \times 6 \mathrm{~cm}$ smooth, firm, non-tender anterior neck mass that moved with deglutition. Rigid endoscopy showed a right supraglottic mass with bulging of the right glottic and subglottic area with a less than 10\% airway opening. (Figure 1A) Both arytenoids were visibly mobile but glottic closure was impaired. (Figure 1B) Tracheostomy and suspension laryngoscopy with biopsy yielded inconclusive results (fibromuscular tissue) and fine needle aspiration cytology (FNAC) of the anterior neck mass only revealed blood and colloid. Contrast computed tomography of the neck showed a well-marginated, hypodense, thickwalled, heterogeneously enhancing mass in the right laryngeal fossa measuring $2.86 \times 1.78 \mathrm{~cm}$ with a larger extension anteriorly measuring $4.66 \times 2.52 \mathrm{~cm}$. Effacement of the epiglottis and aryepiglottic fold was noted. The hyoid and thyroid cartilage were intact and the thyroid gland was normal. (Figure 2A, B)

Because of inconclusive histopathological and cytological results, an incision biopsy of the anterior neck mass was performed. Histopathological evaluation revealed spindle cell mesenchymal proliferation, and immunohistochemical stains showed positive immunoreactivity for CD34, with a weakly positive S-100 and negative SMA, favoring a solitary fibrous tumor. (Figure 3)
Disclosures: The authors signed disclosures that there are no financial or other (including personal) relationships, intellectual passion, political or religious beliefs, and institutional affliations that might lead to a conflict of interest. 


\section{FEATURED GRAND ROUNDS}

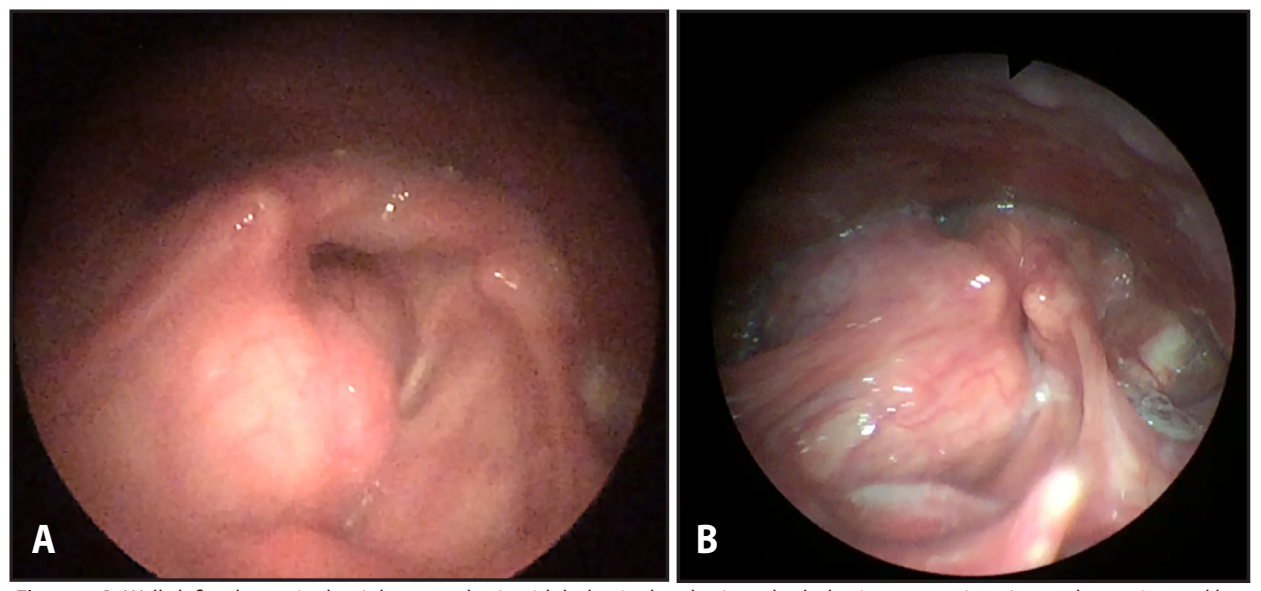

Figure $1 \mathrm{~A}$. Well-defined mass in the right supraglottis with bulge in the glottic and subglottic area causing airway obstruction and less than $10 \%$ airway opening; and B. Impaired glottic closure on adduction with bilaterally mobile arytenoids.

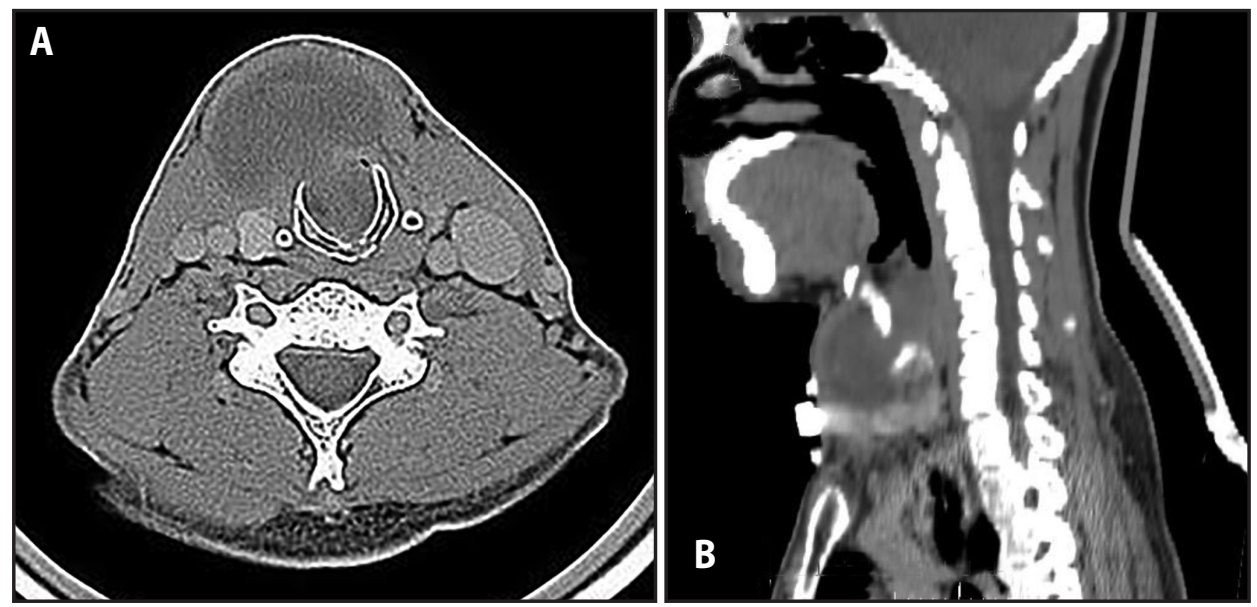

Figure 2 A. Axial and B. Sagittal contrast computed tomography scans reveal a well-marginated, hypodense, thick-walled heterogeneously enhancing mass in the right laryngeal fossa with large extension anteriorly. Note resulting narrowing of the laryngeal airway by about $70 \%$.

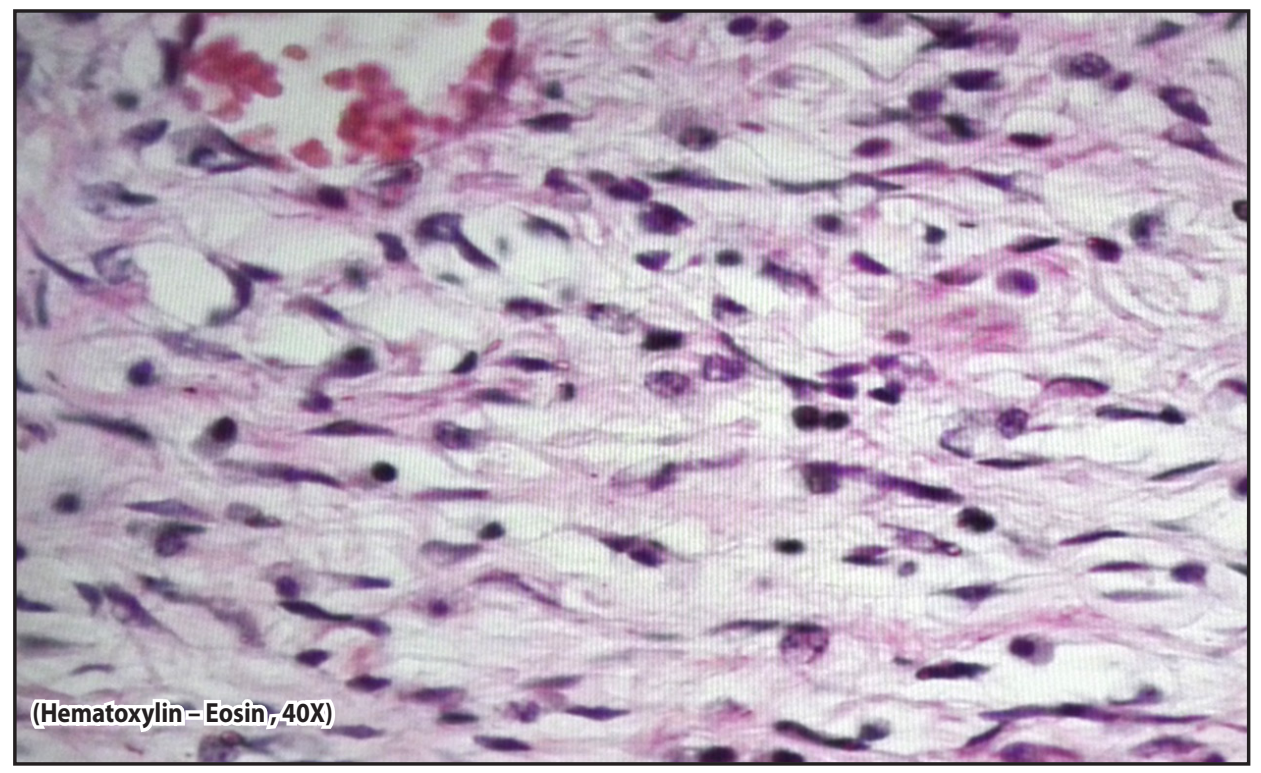

Figure 3. Hematoxylin-Eosin stained histopathologic slide of anterior neck incision biopsy specimen, high power view, 40X showing spindle-cells arranged in a haphazard pattern. 


\section{FEATURED GRAND ROUNDS}

\section{DISCUSSION}

Solitary fibrous tumor is a rare spindle-cell neoplasm, first mentioned and regarded solely as a pleural tumor. ${ }^{1}$ The general incidence of SFT occurs with equal sexual predilection and all age groups are equally affected. ${ }^{2}$ Extrathroracic SFTs have also been documented. Only $6 \%$ of SFT develop in the head and neck and the oral cavity was reported to be the most commonly affected area. ${ }^{3}$ Solitary fibrous tumors of the upper respiratory tract comprise $<0.1 \%$ of all SFTs. ${ }^{4}$ Cases of SFT have also been documented in the paranasal sinuses, nasopharynx and nasal cavity. ${ }^{5}$ There is one reported case of anterior neck SFT in the thyroid ${ }^{7}$ and 2 perithyroidal cases $^{6}$ although it rarely affects the larynx. ${ }^{6}$

Laryngeal SFTs have a male over female predominance. ${ }^{7}$ The subsites frequently involved are the epiglottis, ventricular fold, aryepiglottic fold, vocal fold or the commissure. The clinical presentation of laryngeal SFT's depends on the area involved similar to laryngeal tumors. Those involving the supraglottis present with foreign body sensation, "hot potato" voice, ${ }^{6}$ and dysphagia. ${ }^{8}$ Glottic involvement presents with hoarseness and in late stages (and as is the case with subglottic tumors), there is difficulty of breathing due to obstruction. ${ }^{8}$

Solitary fibrous tumor is generally benign and can be seen as a painless mass that is slow-growing ranging from months to years. ${ }^{1,2,9,10}$ It is difficult to diagnose clinically and other soft tissue tumors (sarcoma, benign fibrous histiocytoma, schwannoma, neurofibroma and fibroma) should be included as differential diagnosis. $29,11,12$

The computed tomography scan in our case showed a well-defined heterogeneously enhancing lesion, with effacement of the arytenoids and epiglottis, displaying the features of a benign soft tissue tumor. Despite extension of the lesion to adjacent structures of the anterior neck, the thyroid and muscles were not invaded and there were no lytic changes of the thyroid cartilage. A benign lesion was highly regarded but a malignant lesion could not be overlooked. It was possible that continuous expansion of the lesion caused the cricothyroid cartilage to efface and allow access of the mass into the right laryngeal mucosa, causing expansion into the inlet and subsequent airway obstruction. On computed tomography scan, benign SFT presents as a dense, welldefined enhancing lesion with expansile growth pattern and no bony erosion or lytic changes, as may be true for other benign tumors of the head and neck-- and other soft tissue tumors could not be easily ruled out. ${ }^{2,11}$ Solitary fibrous tumor is an expansile lesion that can lead to remodeling of adjacent bony structures ${ }^{2}$ and the features of a benign SFT on bone window can be misleading and lead us to disregard any consideration of malignancy. ${ }^{2}$ A malignant SFT can appear to have benign features on $\mathrm{CT}$ scan such as remodeling and only a small foci of bone destruction or lytic changes may be overlooked. ${ }^{2}$ Furthermore, absence of bone remodeling does not entirely rule out malignancy. ${ }^{2}$
A Magnetic Resonance Imaging can also be done for inconclusive CT scan findings. This will show a densely well-circumscribed mass with expansile character and low to moderate enhancement on both $\mathrm{T} 1$ and T2-weighted imaging. ${ }^{12}$ However, we did not perform an MRI in this case.

Gross pathological description of usual SFT is a whitish to grayish, smooth, well-defined lesion. ${ }^{2}$ Histological description shows wide variability of cellularity composed of bland, round to spindle-shaped cells with scanty cytoplasm, and is often associated with stromal hyalinization. ${ }^{4,13}$ The cells are arranged in a haphazard manner, often in a "patternless pattern," no specific cellular pattern or variable cellular pattern with no cytologic atypia. ${ }^{2,45,10,14-16}$ It has collagen bundles with interlaced thin-walled vascular spaces. ${ }^{14,11,16}$ The vascularity of SFT can form a hemangiopericytoma-like pattern hence, it was previously considered a component of hemangiopericytoma. ${ }^{25,10,11,13,17}$ Malignant SFTs have also been reported and histologically display malignant differentiation, such as hypercellularity, cytologic atypia, increased nuclear to cytoplasmic ratio, and mitotically active cells of more than 4 mitosis per $10 \mathrm{HPF} .12,9,13,14,18,19$ There can also be note of tumor necrosis or infiltrative margins in cut sections. These were not observed in our case.

However, the histologic description of benign SFT could also be used to describe other soft tissue tumors, hence, there is no definitive histopathologic picture that may clearly differentiate it from other benign mesenchymal tumors. 'Immunohistochemical reactivity stains are essential to identify SFT from other benign mesenchymal tumor. CD34 is a hematopoietic stem cell and progenitor cell marker ${ }^{20}$ that is a sensitive marker for SFTs. ${ }^{5}$ However, it is also a marker for other mesenchymal stromal cells and epithelial tumors and is a non-specific marker for SFT. ${ }^{5}$ Conversely, $95 \%$ of SFTs consistently stain strongly for CD34. 5,19 There are other reported cases of diagnosed SFT that also have positive reactivity to bcl-2 markers, and commonly lack reactivity for desmin, cytokeratin, and generally negative for actin/smooth muscle actin (SMA).' This could help in the histological differentiation of other soft tissue tumors such as benign fibrous histiocytoma, fibroma and neurogenic tumors.'

In our case, the tumor had a strong reactivity to CD34 and also weak reactivity to SMA. An intensely positive CD34 immunostaining favors a diagnosis of SFT since around $85-100 \%$ of SFT is reactive to CD34. ${ }^{13}$ However, a positive reactivity to CD34 could be a misleading diagnosis since other soft tissue tumors may be positive for both CD34 and SMA. Leiomyosarcoma and schwannoma could also have weakly positive reactivity to CD34 but should also be noted to have a strong reactivity to SMA. Furthermore, SFT may have a consistently positive immunoreactivity to STAT6 immunohistochemistry stains which may 
FEATURED GRAND ROUNDS

be helpful to use as a marker for inconclusive SFT. ${ }^{16}$

The etiology of SFT is still unknown although there is evidence of recurrent NAB2-STAT6 gene fusions integrative sequence due to translocations of the STAT6 gene to NAB2 in SFT's. ${ }^{21}$ Expression of this fusion causes the activation of EGR1 gene, a repressor of transcription pathways which activates the mitogenic pathways of neoplasia. ${ }^{21}$ Detection of NAB2-STAT6 fusions in tumors is strongly associated in the proliferation of SFT's and is an important marker to be considered for an inconclusive immunohistochemical staining result. ${ }^{21}$

About $87 \%$ of SFT's are benign and conservative treatment can be considered. The use of conservative surgical procedures such as $\mathrm{CO} 2$ laser excision could be a treatment option for laryngeal SFT and there was no complication or recurrence reported. ${ }^{5}$ However, it is important to consider that this type of surgical excision will largely depend on the size of the lesion. Furthermore, since studies have shown that both benign and malignant SFT have a relatively high local recurrences of $21 \%$ to $47 \%$, the surgical treatment that should be considered includes radical resection or wide resection with clear margins. ${ }^{14} \mathrm{~A}$ patient diagnosed with SFT of the larynx may need total or partial laryngectomy depending on the affected subsites of the larynx. For malignant SFT that underwent complete resection with clear margins, the reported 10 -year survival rate ranges from $54 \%$ to $89 \%{ }^{14}$ The use of adjuvant chemotherapy is not recommended since most patients will not be responsive. ${ }^{14,21}$ Conversely, radiotherapy can be used for local recurrence or for local control, as well as an option for unresectable tumor although it does not affect overall survival rate. ${ }^{14}$

The incision biopsy result of our patient was considered to be benign solitary fibrous tumor, however, malignancy was still not completely ruled out. Since the mass had already encompassed the laryngeal inlet causing obstruction, wide excision (total laryngectomy) is recommended. Frequent postoperative follow-up will be necessary to monitor spread to, or recurrence in adjacent subsites. The use of radiotherapy may be considered if the final histopathologic result suggests malignancy.

\section{REFERENCES}

1. Alawi F, Stratton D, Freedman PD. Solitary fibrous tumor of the oral soft tissues: clinicopathologic and immunohistochemical study of 16 cases. Am J Surg Pathol. 2001 Jul; 25(7): 900-910. PMID: 11420461.

2. Ganly I, Patel SG, Stambuk HE, Coleman M, Ghossein R, Carlson D, et al. Solitary fibrous tumor of the head and neck: a clinicopathologic and radiologic review. Arch Otolaryngol Head Neck Surg. 2006 May; 132(5): 517-525. DOI: 10.1001/archotol.132.5.517; PMID: 16702568.

3. Girardi F, Marinez B, Girardi M. Solitary Fibrous Tumor of the Larynx: report of two new cases. J Bras Patol Med Lab. 2014 May-Jun; 50(3). DOI: 10.5935/1676-2444.20140020.

4. Barnes L., Everson JW, Reichart P, Sidransky D. (Eds.): World Health Organization: Classification of Tumors. Pathology and Genetics of Head and Neck Tumors. 2005. International Agency for Research on Cancer (IARC) Press: Lyon 2005. p45.

5. Alobid I, Alos L, Maldonado M, Menendez LM, Bernal-Sprekelsen M. Laryngeal solitary fibrous tumor treated with $\mathrm{CO}_{2}$ laser excision: case report. Eur Arch Otorhinolaryngol. $2005 \mathrm{Apr}$; 262(4): 286-288. DOI: 10.1007/s00405-004-0805-1; PMID: 15170575.

6. Villaschi S, Macciomei MC. Solitary fibrous tumor of the perithyroid soft tissue: Report of a case simulating a thyroid nodule. Ann Ital Chir. 1996 Jan-Feb; 67 (1): 89-91. PMID: 8712624

7. Thompson L, Karamurzin Y, Li-cheng M, Kim J. Solitary Fibrous Tumor of the Larynx. Head and Neck Pathol. (2008) 2:67-74. DOI 10.1007/s12105-008-0044-7

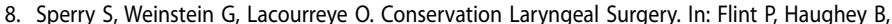
Lund V, Niparko J, Robbins T, Thomas, R, Lesperance M (Eds). Cummings Otolaryngology-Head and Neck Surgery. Sixth Edition. Saunders (Philadelphia). 2015. p. 1674-1698.

9. Bruzzone A, Varaldo M, Ferrarazzo C, Tunesi G, Mencoboni M. Solitary Fibrous Tumor. Rare Tumors. 2010; 2:e64.

10. Gold JS, Antonescu CR, Hajdu C, Ferrone CR, Hussain M, Lewis JJ, et al. Clinicopathologic correlates of solitary fibrous tumors. Cancer. 2002 Feb 15; 94(4): 1057-68. DOI 10.1002/ cncr.10328; PMID: 11920476

11. Hasegawa T, Hirose T, Seki K, Yang P, Sano T. Solitary fibrous tumor of the soft tissue: an immunohistochemical and ultrastructural study. Am J Clin Pathol. 1996 Sep; 106(3):325-31. PMID: 8816589.

12. Kunzel J, Hainz M, Ziebart T, Pitz S, Ihler F, Strieth S, et al. Head and neck solitary fibrous tumors: a rare and challenging entity. Eur Arch Otorhinolaryngol. 2016 Jun; 273(6): 1589-1598. DOI: 10.1007/s00405-015-3670-1; PMID: 26026772.

13. Geramizadeh B, Marzban M, Churg A. Role of Immunohistochemistry in the Diagnosis of Solitary Fibrous Tumor, a Review. Iran J Pathol. 2016 Summer; 11(3):195-203. PMID: 27799967 PMCID: PMC507945

14. DeVito N, Henderson E, Han G, Reed D, Bui MM, Lavey R, et al. Clinical Characteristics and Outcomes for Solitary Fibrous Tumor (SFT): A Single Center Experience. PLoS ONE. 2015 Oct; 10 (10): e0140362. DOI: 10.1371/journal.pone.0140362; PMID: 26469269 PMCID: PMC4607370.

15. Fletcher CD. Distinctive soft tissue tumors of the head and neck. Mod Pathol. 2002 Mar; 15(3):324-330. DOI: 10.1038/modpathol.3880526; PMID: 11904345.

16. Topaloglu O, Ucan B, Demirci T, Sayki AM, Saylam G, Onder E, et al. Solitary fibrous tumor of neck mimicking cold thyroid nodule in 99m tc thyroid scintigraphy. Case Rep Endocrinol. 2013 2013: 805745. DOI: 10.1155/2013/805745; PMID: 24194989 PMCID: PMC3806406.

17. Fletcher CD, Path FRC. Distinctive soft tissue tumors of the head and neck. Mod Pathol $2002 \mathrm{Mar}$ 15(3): 324-330. DOI: 10.1038/modpathol.3880526; PMID: 11904345

18. Dotto JE, Ahrens W, Lesnik DJ, Kowalski D, Sasaki C, Flynn S. Solitary fibrous tumor of the larynx: a case report and review of literature. Arch Pathol Lab Med. 2006 Feb; 130(2):213-216. DOI: 10.1043/1543-2165(2006)130[213:SFTOTL]2.0.CO;2; PMID: 16454566.

19. Doyle LA, Vivero M, Fletcher CD, Mertens F, Hornick JL. Nuclear expression of STAT6 distinguishes solitary fibrous tumor from histologic mimics. Mod Pathol. 2014 Mar; 27(3): 390395. DOI: 10.1038/modpathol.2013.164; PMID: 24030747.

20. Sidney LE, Branch MJ, Dunphy SE, Dua HS, Hopkinson A. Concise review: evidence for CD34 as a common marker for diverse progenitors. Stem Cells. 2014 Jun; 32(6): 1380-1389. DOI: 10.1002/ stem.1661; PMID: 24497003 PMCID: PMC4260088.

21. Robinson DR, Wu YM, Kalyana-Sundaram S, Cao X, Lonigro RJ, Sung YS, et al. Identification of recurrent NAB2-STAT6 gene fusions in solitary fibrous tumor. Nat Genet. 2013 Feb; 45(2): 180 185. DOI: 10.1038/ng.2509; PMID: 23313952 PMCID: PMC3654808.

22. Langman G. Solitary fibrous tumor: A pathological enigma and clinical dilemma. J Thorac Dis. 2011 Jun; 3(2): 86-87. DOI: 10.3978/j.issn.2072-1439.2011.03.04; PMID: 22263070 PMCID: PMC3256512. 